\title{
À propos du site de la vallée de Mouéara et des combats du Cap Goulvain en janvier 1879 en Nouvelle-Calédonie
}

Alain Saussol

\section{(2) OpenEdition}

Journals

Édition électronique

URL : http://journals.openedition.org/jso/1340

DOI : $10.4000 /$ jso. 1340

ISSN : $1760-7256$

Éditeur

Société des océanistes

Édition imprimée

Date de publication : 1 décembre 2003

Pagination : 317-324

ISSN : 0300-953x

\section{Référence électronique}

Alain Saussol, «À propos du site de la vallée de Mouéara et des combats du Cap Goulvain en janvier 1879 en Nouvelle-Calédonie », Journal de la Société des Océanistes [En ligne], 117 | Année 2003-2, mis en ligne le 22 mai 2008, consulté le 15 septembre 2020. URL : http://journals.openedition.org/jso/ 1340

\section{(c) Tous droits réservés}




\section{Mouéara, la « forteresse » oubliée}

Le JSO publie ici dans les miscellanées, deux notes illustrées à propos de la «forteresse » de Mouéara, qui fut le lieu de combats importants à la fin de la "Grande révolte » de 1878. La première, d'Alain Saussol, retrace les combats et la redécouverte du site en précisant sur une carte la localisation de la "forteresse ». La seconde, de Claude Cornet, présente le site tel qu'elle a pu le voir aujourd'hui et précise l'emplacement de quelques vestiges avec carte et photographies.

L' 'intérêt exceptionnel de ce site » impose que soient prises au plus vite des mesures de protection et de conservation. Espérons que la publication de ces deux notes y contribue.

\section{À propos du site de la vallée de Mouéara et des combats du Cap Goulvain en janvier 1879 en Nouvelle-Calédonie}

par

\section{Alain SAUSSOL*}

Le hasard d'un travail sur la grande insurrection kanak de 1878 en Nouvelle-Calédonie m'a incité à rechercher les lieux précis où se déroulèrent ces événements, de façon à pouvoir les intégrer dans leur contexte environnemental. C'est ainsi que je fus conduit à m'intéresser aux combats «du Cap Goulvain », curieusement estompés dans la mémoire calédonienne alors qu'ils représentent les combats les plus durs et les plus sanglants de la grande insurrection ${ }^{1}$.

Cette note se propose de rappeler ce que furent ces combats autour de ce réduit que les officiers qualifièrent de "forteresse » kanak et de relater comment le site en fut retrouvé. Mais elle entend surtout attirer l'attention sur l'urgente nécessité de prendre en compte et de protéger ce mémorial encore intact de l'histoire calédonienne.

\section{Les combats du Cap Goulvain (3-12 janvier 1879)}

L'action se développe au début du mois de janvier 1879 dans la région de Bourail. L'insurrection kanak, commencée en juin 1878 par les massacres de la Foa et de Bouloupari, étendue en septembre aux régions de Poya et de Bourail, tire alors sur sa fin. Les dernières bandes rebelles se cachent dans les solitudes éloignées

1. Il est possible que la mémoire mélanésienne en ait conservé le souvenir. Il y aurait, en Nouvelle-Calédonie, un long et fructueux travail à mener à partir de cette mémoire ignorée pour l'ensemble de l'insurrection, comme nous avons pu l'expérimenter avec le maire de Thio, Joël Dauphiné et Jerry Delathière à Borendi ainsi qu'auprès du clan Moindou, dans la haute vallée de Koua.

* Historien, université de Montpellier, alain.saussol@wanadoo.fr 


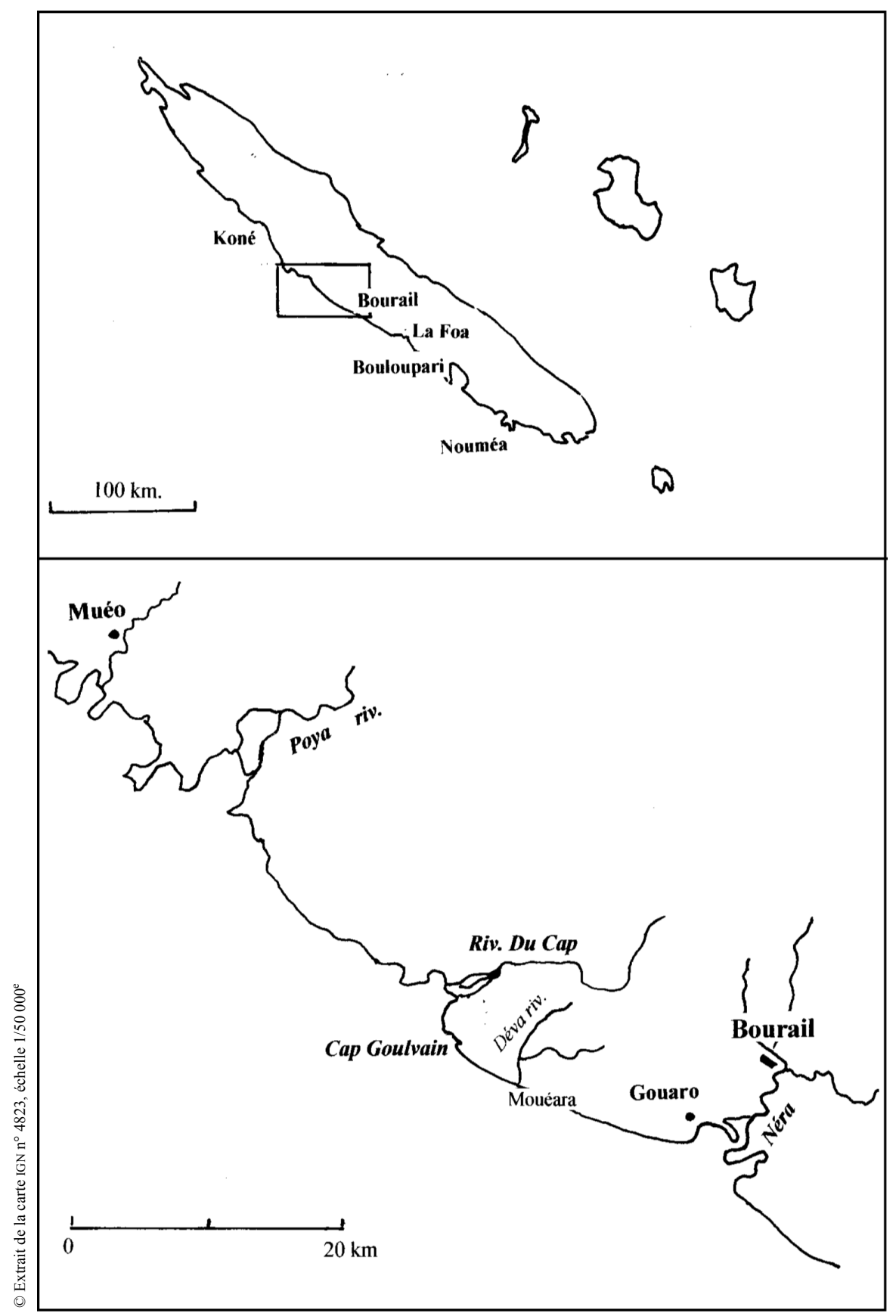

FIG. 1 - Bourail, localisation et carte d'ensemble

des villages européens. Mais l'insécurité règne toujours aux abords des centres de colonisation, interdisant toute reprise d'une vie normale. À Bourail où se groupent quelques colons réfugiés, chacun pense qu'il est temps d'en finir. Le 2 janvier 1879, le bourg pénitentiaire vient de fêter, sous la pluie, un triste nouvel an et attend la visite imminente du gouverneur Olry.
À l'aube du 3 janvier, à Gouaro, au bord de la baie de Bourail, un détachement commandé par le lieutenant Vaux-Martin², renforcé par cinquante auxiliaires de la tribu de $\mathrm{Ni}$, quitte le poste militaire pour une patrouille de routine. Un quart d'heure plus tard, ils surprennent un groupe de Kanaks révoltés venus chercher des provisions dans les cultures des concessionnaires. 
Une poursuite s'engage sur dix-huit kilomètres le long de la plaine côtière allant de Poé au Cap Goulvain ${ }^{3}$.

Chemin faisant, au bord d'une "grande mangrove », la colonne tombe sur « de grands campements » dont les occupants parviennent à fuir, à l'exception d'une femme qui est capturée. La poursuite prend fin quelques kilomètres plus loin, devant la "vallée de Mouéara ", où les fuyards se sont retranchés « dans une montagne, couverte de grands bois et parsemée de rochers $»$.

Vaux-Martin tente d'enlever la position, mais il essuie «nombre de coups de fusil». Les auxiliaires qui n'ont que leurs armes traditionnelles, serrés de près par les rebelles, ont deux tués et trois blessés par balles. Surpris par ce déploiement inhabituel d'armes à feu, le lieutenant $n$ 'insiste pas. Il juge prudent de faire demitour, quitte à revenir en force plus tard. Le détachement rentre donc à Gouaro, ramenant la femme prise au campement de la mangrove.

Le soir même, Vaux-Martin l'interroge. La prisonnière lui apprend qu'il y a là-bas la plupart des insurgés de Bourail et de Poya (ceux de Porwy, de Néra, de Nékou, de Ouanho et de Nandaï), avec les armes prises sur la station Houdaille (des carabines Snider), et que la vallée est fortifiée (ce que l'officier n'avait, semble-til, pas remarqué). Fort de cette information capitale, le lieutenant rédige un rapport pour le commandant de Maussion qui représente l'autorité militaire à Bourail. Toute la journée, le bourg s'était trouvé animé par les festivités organisées pour la visite du gouverneur Olry, qui avait débarqué le matin même à Gouaro.

Le lendemain 4 janvier, de bonne heure, Bourail apprend le violent accrochage survenu la veille au Cap Goulvain. Cette nouvelle éclipse, dans les commentaires, la visite du gouverneur. À midi, venant de Gouaro, arrivent les auxiliaires de Ni portant leurs morts ficelés à de grandes perches et enveloppés d'écorces, pour les ramener dans leurs villages. Leurs chefs sont reçus par Olry. Sans doute se plaignent-ils d'être moins bien armés que les rebelles car le gouverneur en retient quarante qu'il décide, mesure très inhabituelle, de doter de « chassepots », les fusils de guerre de l'époque.

Pendant ce temps, Maussion, qui a lu le rapport de Vaux-Martin, comprend qu'il y a un coup à tenter qui peut sonner l'hallali de l'insurrection. Il n'y a pas de temps à perdre. Avec l'aval du gouverneur, il prépare sur le champ une seconde expédition qu'il commandera en personne. La colonne comprend cinquante soldats d'infanterie de marine, les quarante auxiliaires Honrôés ${ }^{4}$ dotés de fusils et deux autres officiers, Vaux-Martin et le sous-lieutenant Rochel. Le jour même, à dix-sept heures, tous prennent la route pour Gouaro où ils passeront la nuit. Le gouverneur reste à Bourail et poursuit son programme de visite.

Le dimanche $\mathbf{5}$ janvier, de bon matin, la colonne Maussion quitte Gouaro. Il pleut. À l'approche de la vallée de Mouéara, elle est repérée par des guetteurs kanak perchés sur les crêtes. Il n'y aura pas d'effet de surprise. La colonne arrive enfin devant cette « vallée encaissée entre deux croupes » et formant « un ovale très allongé », « pleine de rochers d'un effet très pittoresque ». La vallée est boisée, " couverte d'arbres et de brousses qui en font un fouillis inextricable ». Sur ses lisières, « les Canaques avaient construit une série de petits murs en grosses pierres disposés d'une façon très intelligente et qui leur permettaient de voir et de tirer, tout à leur aise, en restant à l'abri ».

Pour cerner la vallée, Maussion dédouble sa colonne. Il en confie une moitié à Vaux-Martin avec charge d'occuper la crête qui la ferme vers l'ouest (Nindouri) et garde l'autre avec le souslieutenant Rochel pour tenir celle de l'est (partant du pic Caillou). Chaque détachement gravit son contrefort et en suit la crête en restant sur le versant externe pour se mettre à l'abri des tirs des Kanaks. Maussion et sa troupe arrivent ainsi à deux cents mètres au-dessus des positions tenues par les rebelles qui, en contrebas, sont pris à revers.

Pendant ce temps, le lieutenant Vaux-Martin poursuit sa progression et atteint bientôt « le point culminant » (sans doute le pic Noir ou le

3. Je me suis un moment demandé si cet épisode n'était pas un guet-apens tendu à la troupe, ces « voleurs de patates » se laissant surprendre et poursuivre, en évitant de semer les soldats, pour les entraîner à l'endroit préalablement choisi par eux pour leur infliger une correction. (Une répétition en quelque sorte du guet-apens tendu à La Foa au colonel Gally Passebosc, très probablement attiré sur le lieu de son exécution par le chef Naïna, sectionnant à cet endroit le fil du télégraphe.) Toutefois, rien ne permet de l'affirmer ici.

4. «Honrôés » (encore orthographié « Orôwé » ou « Orôé »), terme utilisé par les Européens pour désigner les gens de la montagne (tribus de $\mathrm{Ni}$ et de Quicoué) du haut pays de Bourail. Ces montagnards, après avoir jadis combattu les Français, étaient devenus en 1878 leurs alliés. 
sommet Déva). Il place ses hommes en position de tir et, par des feux de peloton, déloge les rebelles embusqués plus bas dans les rochers, les contraignant à se replier vers le fond de la vallée. Du même coup, il leur coupe toute possibilité de retraite par l'amont. Cela fait, il disperse ses hommes en tirailleurs et commence à dévaler le versant, fouillant le terrain et refoulant les Kanaks de plus en plus bas.

C'est alors que Maussion, à son tour, déploie ses hommes sur la crête d'en face et ordonne le second mouvement de l'attaque. Dès qu'ils les aperçoivent, les Mélanésiens ouvrent le feu dans leur direction. Leur fusillade est si nourrie que le commandant juge qu'ils consomment là une grande partie de leurs cartouches. Ses hommes et leurs alliés Honrôés, qui dominent les postes de tir des rebelles, répliquent. Puis, se déployant à leur tour en tirailleurs, ils cernent les rochers et les "fortifications » plus nombreuses sur ce versant et, à coups de chassepot, contraignent les occupants à décrocher.

L'une après l'autre, les positions des rebelles sont ainsi dégagées, tandis que leurs défenseurs reculent vers le bas. Dans sa progression, le détachement trouve des traces de sang et des "débris humains » prouvant que les insurgés subissent des pertes. La troupe aussi a perdu un homme, le soldat Deschavannes, atteint de deux balles en pleine poitrine au début de l'attaque.

Les heures passent dans la chaleur lourde et dans l'excitation du combat. À mesure que l'on descend, la résistance kanak se fait plus vive. À quinze heures, Maussion fait sonner l'ordre de ralliement pour Vaux-Martin et un peu plus tard, les deux détachements font leur jonction. Vers seize heures, alors que s'abat une pluie violente, on arrive devant les derniers retranchements de la vallée. Invisibles, tapis derrière leurs murs et leurs rochers, les rebelles se défendent toujours avec l'énergie du désespoir. Les soldats sont fatigués par la longue marche matinale, la journée de combat, la chaleur oppressante et la pluie qui s'en mêle. Leur vigilance se relâche-t-elle? Le fait est que la colonne va subir là ses plus lourdes pertes. Deux hommes, Chauvet et Astoury, qui suivaient Maussion, sont blessés par balles. Le commandant luimême est atteint à l'épaule tandis qu'une autre balle lui érafle le front. Peu après, deux autres soldats sont touchés aux genoux. Pire, le souslieutenant Rochel reçoit une balle en pleine poitrine. Un auxiliaire kanak est tué. En tout, six guerriers de $\mathrm{Ni}$ ont été tués dans la journée (dont l'un, peut-être, par méprise d'un soldat). Comparé aux opérations militaires ordinaires, c'est une hécatombe. L'ampleur inhabituelle des pertes, surtout la grave blessure de Rochel, qui mourra, une heure plus tard, étouffé par son sang, convainc Maussion d'arrêter le combat.

C'est une sage décision. La troupe est harassée, le soir approche. Les six victimes européennes (les morts et blessés) vont mobiliser quinze hommes pour les ramener, portant à vingt et un l'effectif de soldats mis hors de combat, presque la moitié du détachement. Il en va de même pour les auxiliaires qui tiennent aussi à ramener leurs morts. Mieux vaut donc s'éloigner de ces parages et se rapprocher du poste avant la nuit.

Le feu cesse. La troupe se replie. La colonne reconstituée, meurtrie, se remet en ordre de marche et, sous la pluie battante, sans être inquiétée par les insurgés, prend lentement le chemin du retour. Il est neuf heures du soir et il fait nuit depuis longtemps quand, épuisée, elle arrive à Gouaro. L'ambiance n'est pas à la fête. Le lorrain Rochel était un bon officier " plein d'avenir », estimé de ses chefs et de ses hommes, apprécié par le Père Lecouteur, curé de Bourail (qui rappelle qu'on trouva sous sa chemise un scapulaire ensanglanté). À peine arrivés, on embarque les morts et les blessés dans une baleinière pour les évacuer sur Bourail en remontant la Néra. Ils y parviendront dans la nuit pour être conduits à l'hôpital.

Le lendemain, la colonne expéditionnaire regagne Bourail, par la route. Les Honrôés, préalablement désarmés, rejoignent leurs villages en emportant leurs morts.

Le mardi 7 janvier, Bourail est en deuil. L'après-midi, dans le petit cimetière au flanc de la colline, on enterre le sous-lieutenant Rochel et le soldat Deschavannes, côte à côte dans la même fosse. Toute la garnison est là, officiers, surveillants militaires, fonctionnaires. Un détachement rend les honneurs et exécute une salve.

Il n'est pas question, toutefois, de rester sur cet échec. Les jours suivants, on prépare donc une troisième expédition. On peut, d'un coup, terminer l'insurrection. Celui qui emportera la position, en écrasant les derniers rebelles, sera le vainqueur de la guerre. Certes, l'affaire s'annonce rude car les Kanaks paraissent déterminés et disposent, croit-on, d'une soixantaine de fusils. Mais la victoire est au bout et la gloire avec ! 


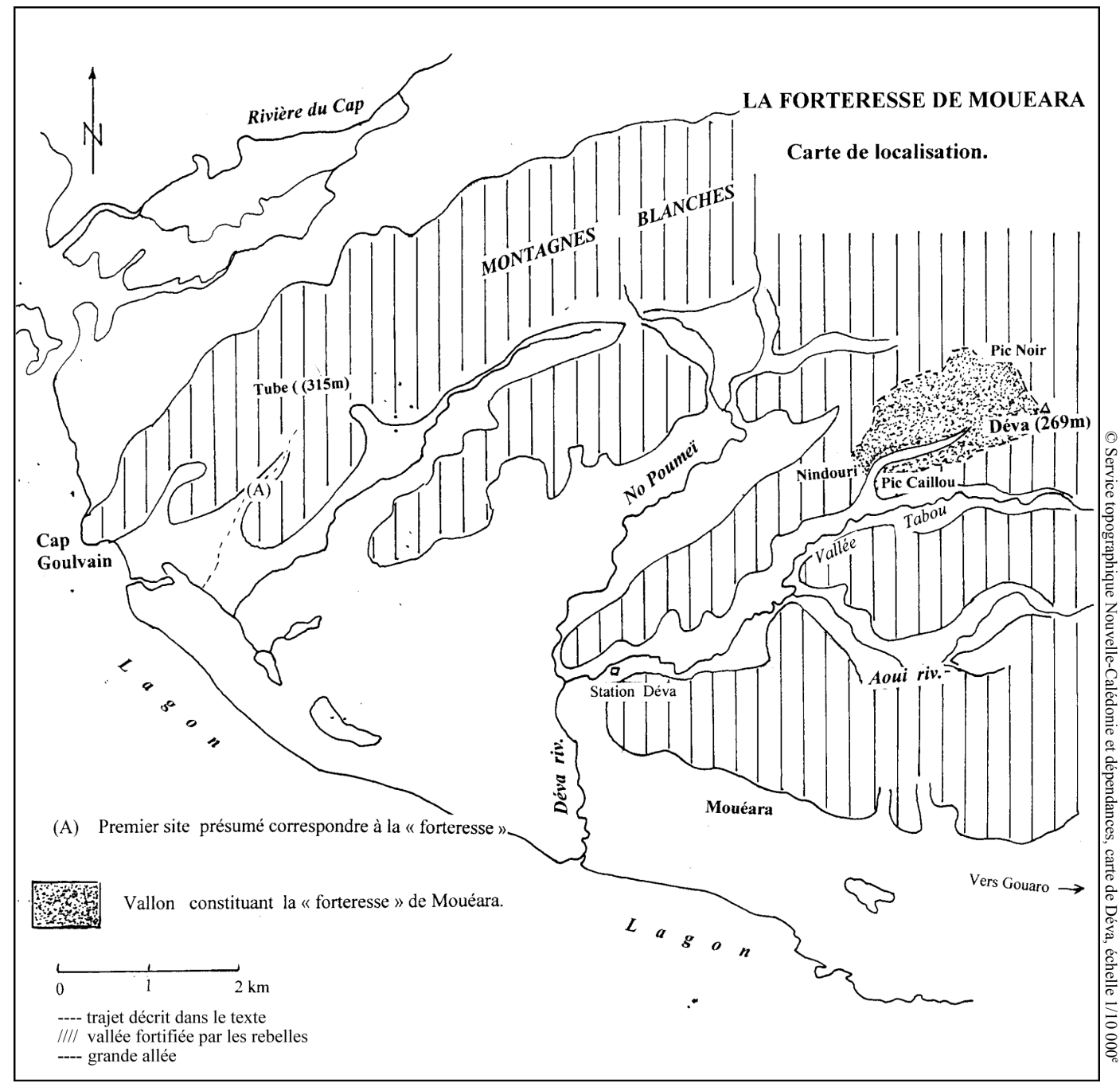

FIG. 2 - La forteresse de Mouéara

Coiffant Maussion, le colonel Wendling, commandant supérieur des troupes en NouvelleCalédonie, prend en personne le commandement de cette nouvelle expédition. Wendling exclut un nouvel échec. Le commandement militaire, qui vient de perdre un officier, n'entend pas perdre la face. Il mobilise donc tout ce que Bourail compte de combattants potentiels : soldats d'infanterie, artilleurs avec leur obusier, condamnés armés, Arabes, sans compter les supplétifs Honrôés qui rallient Bourail et auxquels on rend leurs fusils. Des renforts de troupes appelés de Muéo et de Koné débarquent le 10 janvier à Gouaro.

Le samedi $\mathbf{1 1}$ janvier à midi, le colonel et sa troupe quittent Bourail. Le Père Lecouteur les suit à cheval. Le brave curé, redoutant une hécatombe, a demandé à les accompagner pour assister les mourants. C'est dire combien la violence de la tentative précédente a frappé les esprits. En fin d'après-midi, c'est le regroupement général de toutes les forces à Gouaro où est prévu le bivouac.

Le dimanche $\mathbf{1 2}$ janvier, à trois heures du matin, la colonne Wendling s'ébranle. Elle comprend plus de trois cents hommes de toutes armes sans compter les alliés kanak. La marche est lente. Dans le sol détrempé de la plaine, on s'enfonce parfois jusqu'aux genoux.

Il est dix heures quand on arrive devant le réduit de Mouéara. Répétant la manœuvre de Maussion, Wendling entreprend de cerner la vallée par les crêtes et occupe les positions. Cette fois, on n'a pas aperçu de guetteur. Dans la vallée rien ne bouge. Pour tester l'adversaire, le colonel décide de donner du canon. L'obusier 
est hissé sur « un mamelon très raide ». L'artilleur Millet le pointe. Deux obus sont tirés contre des rochers sans provoquer de réaction. Bizarre. Le colonel se dit qu'ils ont dû déguerpir. Mais pour en avoir le cœur net, il ordonne au clairon de sonner l'en-avant.

La troupe avance donc, alliés en tête poussant leur cri de guerre. Les hommes s'approchent prudemment des rochers, visitent les grottes. Le site est désert. On fouille, malgré tout, la vallée. Bientôt, il faut se rendre à l'évidence : les rebelles ont décampé. C'est pour le colonel une cruelle déception. Il n'a plus qu'à ordonner une halte casse-croûte. Le menu est frugal : un morceau de pain sec.

Pendant ce temps, les alliés continuent de battre la brousse autour du Cap Goulvain à la recherche des fugitifs. Près de la rivière du Cap, ils découvrent, dans les alluvions, de nombreuses traces de pas, révélant que les rebelles avaient fui en direction de Poya. On saura plus tard (par une prisonnière capturée le 20 janvier), que les défenseurs de la "forteresse » de Mouéara avaient quitté le site au soir du 5 janvier, juste après la retraite de Maussion, sans ramasser leurs cadavres mais « en emportant un grand nombre de blessés sur des brancards improvisés », et que "leurs derniers coups de feu avaient été comme un coup de désespoir $»$.

Pour l'heure, il ne reste à Wendling, dépité, qu'à prendre le chemin du retour. À son habitude, le colonel, à pied, marche en tête de la colonne. Vers dix-sept heures, " harassée de fatigue et couverte de boue ", celle-ci arrive à Gouaro où elle passe la nuit. Seuls l'obusier et ses convoyeurs sont embarqués le soir même dans une baleinière pour Bourail, où ils parviennent vers minuit.

Le lendemain, 13 janvier, l'expédition Wendling rentre à Bourail à pied. Le colonel rend compte au gouverneur. Le Père Lecouteur, qui a suivi l'expédition de loin, s'autorise un commentaire teinté d'ironie :

« Les Canaques ont dû rire beaucoup en voyant ce déploiement de forces militaires et ce siège en règle d'un rempart naturel, n'ayant pour défenseur que les chèvres de la montagne $»$.

Sur ce coup manqué s'achève donc l'épisode des combats du Cap Goulvain, dits aussi de la «vallée de Mouéara ». L'insurrection mori- bonde va perdurer encore quelques semaines avant le retour au calme.

L'enchaînement des événements qui ont marqué cet épisode, ayant pu être ainsi rétabli, il restait à localiser cette "forteresse » kanak devenue, pour les historiens, avec le temps et l'oubli, une sorte de mythe.

\section{À la recherche de la vallée perdue}

Pour la retrouver, on disposait de peu d'éléments. D'abord ces combats étaient donnés comme situés « au Cap Goulvain ». Quelques sources précisaient qu'ils s'étaient déroulés dans une vallée dite "de Mouéara" (nom absent des cartes IGN et du Service topographique, dont je disposais).

On savait qu'ils avaient eu lieu à trente-cinq kilomètres de Bourail. C'était une donnée crédible car les militaires de ce temps, rompus à la marche, savaient évaluer avec précision le kilométrage d'une étape. En l'occurrence, cette distance correspond exactement à celle de Bourail au Cap Goulvain, via Gouaro en longeant la plaine côtière. On pouvait en déduire que c'était à proximité immédiate de l'éperon du Cap que devait se trouvait le fameux site. Mais où exactement?

Pour tenter d'y répondre, il y avait les descriptions, peu précises, des rapports rédigés par les officiers et repris par le Gouverneur, ainsi que celles du journal de marche d'un artilleur'. Outre leurs indications topographiques, ces diverses sources donnaient d'utiles précisions sur le déroulement des combats permettant quelques déductions quant à leur contexte géographique. On savait, par exemple, que la vallée où s'étaient cachés les rebelles avait pu être cernée, depuis les crêtes encadrantes, par quarante soldats et cinquante auxiliaires. C'est dire qu'elle devait être assez courte (guère plus d'un kilomètre).

Sur de tels critères, à partir de la vieille édition de la carte IGN au $1 / 50000^{\mathrm{e}}$ (feuille de Bourail), je retins comme plausible un grand vallon de forme vaguement ovale, ouvert dans le flanc sud des montagnes Blanches, tout près du promontoire du Cap Goulvain. Certes, ce vallon ne correspondait pas idéalement aux éléments décrits par les militaires. Sans être sur place, il n'était pas possible de savoir si son creux recélait une source d'eau potable, indispensable pour 
un campement (mais on pouvait supposer que la proximité de l'embouchure de la rivière du Cap permettait, au pire, d'y pallier). C'est dire combien cette première hypothèse restait aléatoire. Mais aucun autre site, au voisinage immédiat du Cap Goulvain, ne semblait convenir.

Pour la vérifier, sur le conseil de Louis-José Barbançon (historien, bon connaisseur de la région de Bourail), je pris contact avec Claude Cornet, qui venait de publier un livre sur l'insurrection de 1878, agrémenté de photos prises sur le terrain, montrant qu'elle s'intéressait aussi aux lieux. En mars 2002, je lui écrivis donc pour lui soumettre mon hypothèse et avoir son avis. Elle avait l'avantage de connaître de visu le terrain (et de posséder l'édition récente de la feuille IGN de Bourail, enrichie d'une abondante nomenclature d'anciens noms de lieux !).

Dans sa réponse, Madame Cornet me fit remarquer qu'un lieu nommé « Mouéara » était indiqué au bord de mer, près de l'embouchure de la Déva. Elle supposait donc que la vallée des affrontements pouvait correspondre à la vallée Tabou où se trouvaient, disait-elle, d'anciennes traces d'habitats kanak.

Cette vallée me semblait trop vaste pour avoir pu être cernée par une centaine d'hommes. Par contre, j'étais très étonné d'apprendre que Mouéara était au bord de mer, toutes les descriptions décrivant le site comme enchâssé dans la montagne ou dans de hautes collines, donc comme un site d'arrière-pays. Pourtant cette information allait s'avérer décisive.

En effet, en examinant attentivement les parages de la vallée de la Déva, mon attention fut attirée par un étroit ravin affluent, de dimensions modestes, très encaissé entre des pentes escarpées, nanti d'un ruisseau pérenne. Ce ravin correspondait parfaitement aux bribes descriptives dont je disposais. J'eus la conviction que l'endroit que nous cherchions était là, s'ouvrant entre les deux pitons nommés Nindouri et Bwé Ané (ou pic Caillou) et remontant vers la crête boisée qui relie le pic Noir au sommet Déva. Argument supplémentaire, je constatais que cet endroit se trouvait, lui aussi, via Gouaro, à la même distance de Bourail que le Cap Goulvain.

Quant au site nommé Mouéara localisé sur le bord de mer près de l'embouchure de la Déva et d'une mangrove, il devait correspondre à ce grand campement surpris par Vaux-Martin le
3 janvier. Par extension, l'officier avait dû donner à la petite vallée fortifiée située à quelques kilomètres de là, le nom de "vallée de Mouéara» au sens de "vallée proche de Mouéara », Mouéara étant originellement un site côtier. Cette interprétation, conforme à toutes les données connues, semblait cohérente. J'en fis part à Madame Cornet qui, avec son frère Jacques Dolbeau, éleveur au Cap, résolut de se rendre sur le terrain pour voir ce qu'il en était.

C'est Jacques Dolbeau qui, depuis sa station proche, fit la première visite le 26 mai 2002. Il en revint enthousiaste, ayant découvert de longs murs bâtis de grosses pierres, des murets en croissant et des ossements humains épars. Cette trouvaille paraissait valider le site, les restes humains, dispersés sans sépulture, étant manifestement ceux des guerriers kanak tués en défendant la position, le 5 janvier 1879 .

Cette émouvante découverte concluait une lointaine et fructueuse collaboration à laquelle nous devions, ensemble, le succès de notre quête. Les explorations suivantes furent effectuées par Claude Cornet qui, avec son mari, parcourut le site, inventoriant de nouveaux indices et prenant de nombreux clichés ${ }^{6}$.

\section{Un site à préserver}

On doit, en conclusion, souligner l'intérêt exceptionnel de ce site qui reste celui du plus haut fait d'armes mélanésien de l'insurrection de 1878 et un haut lieu partagé de l'histoire calédonienne. Ce mémorial de la résistance kanak, sacralisé par ses morts, fut aussi le champ d'un épisode sanglant de la longue traque menée par l'infanterie de marine contre les bandes rebelles. Face aux insurgés, un soldat, huit auxiliaires mélanésiens et un jeune officier y trouvèrent aussi la mort.

Ce domaine est, par chance, resté à ce jour intact, quasiment dans l'état où il se trouvait en 1879. Le risque serait qu'après avoir été épargné plus d'un siècle par son isolement, le site de la «forteresse» soit aujourd'hui stupidement détruit. Dans un contexte de spéculation immobilière et de péri-urbanisation galopante, le danger est réel.

Ce site, niché dans l'ancienne station Déva, appartient au domaine public de la province Sud, donc à tous ses habitants. Mais, s'ils sont

6. Dans la note qui suit ce texte, ils décrivent le site tel qu'ils l'ont vu et localisent sur fond de plan quelques-uns de ses vestiges. 
les premiers concernés, l'enjeu dépasse une seule province. Il concerne l'ensemble du territoire calédonien impliquant tous ses citoyens quelle que soit leur origine. Mouéara appartient à leur mémoire commune, c'est leur héritage à tous et c'est à eux que s'adresse ce message. Il incombe à leurs représentants, que sont les auto- rités provinciales, mais aussi territoriales et de l'État, de mettre en œuvre les mesures de sauvegarde ou de protection qui s'imposent, par respect pour les hommes qui y ont mêlé leur sang. Mais aussi, au nom de la double mémoire kanak et calédonienne, de la préservation du patrimoine et des fondements identitaires du pays.

\section{Le site de Mouéara}

par

\section{Claude CORNET*}

Alors que je terminais mon ouvrage $L a$ Grande révolte - 1878, je m'étais déjà rendue sur tous les lieux d'affrontements pour bien assimiler leur configuration; il me restait à situer l'endroit où les derniers combats s'étaient déroulés dans les environs du Cap Goulvain. Cette région est brièvement citée par Charles Lemire lors de ses comptes rendus hebdomadaires. Mais, dans des espaces immenses, succession de propriétés privées, où l'accès est donc réglementé, trouver l'emplacement précis où se sont affrontés les derniers rebelles et les troupes françaises était une gageure.

C'est sur la carte IGN au $1 / 50000^{\mathrm{e}}$ de la région de Bourail, mise à jour en 1990, que j'ai trouvé les premières indications : deux lieux, l'un au bord de mer, Mouéara, l'autre dénommé mont Rochel, un peu en amont. J'acquis alors la conviction que Gouaro-Déva était la région dont parlait Charles Lemire dans ses chroniques du Moniteur de la Nouvelle-Calédonie les 8 et 22 janvier 1879 . C'est là que le souslieutenant Rochel fut tué par les insurgés le 5 janvier 1879 et où son capitaine fut blessé sans gravité. La femme du chef de Bouirou, prisonnière, avait dit «qu'à Mouéara les rebelles se sont enfuis pour enterrer leurs morts. Ils ont emporté sur des brancards leurs nombreux blessés ». À Gouaro, elle déclarera au souslieutenant de Vaux-Martin que « la plupart des insurgés de Bourail et Poya se sont réfugiés dans la vallée qui est fortifiée... »

Je connaissais un site au-dessus du marais de Déva (Mouéara) abritant un cimetière kanak où de très nombreux crânes sont dissimulés sous de grands rochers. Ces lieux, qui correspondent bien aux descriptions faites en 1879 par les militaires, sont grandioses, impressionnants ; j'en possédais de nombreuses photos montrant leur étendue, depuis le sommet du Nérouaou jusqu'au bord de mer. Lors de ces anciennes excursions, j'avais déjà remarqué, au sud de ce pic et à l'extrémité est de la grande vallée nommée aujourd'hui "vallée Tabou », des tertres d'anciennes cases et d'abondants débris de coquilles en surface, attestant d'une présence humaine importante datant de plusieurs siècles, le tout malheureusement dégradé par l'érosion et les animaux sauvages.

En octobre 2000, je publie mon livre illustré des photographies des différents endroits où les affrontements se sont déroulés. C'est en mars 2002 que le professeur Saussol, qui a récemment eu connaissance de mon travail, prend

* Institutrice à la retraite, Nouvelle-Calédonie, gccornet@lagoon.nc 